\title{
Minor-merger-driven growth of early-type galaxies over the last 8 billion years
}

\author{
S. Kaviraj ${ }^{1}$, R. M. Crockett $^{2}$, J. Silk ${ }^{2}$, R. S Ellis ${ }^{3}$, S. K. Yi ${ }^{4}$, \\ R. W. O'Connell ${ }^{5}$, R. Windhorst ${ }^{6}$ and B. C. Whitmore ${ }^{7}$ \\ ${ }^{1}$ Blackett Laboratory, Imperial College London, London SW7 2AZ \\ ${ }^{2}$ Dept. of Physics, Denys Wilkinson Building, Keble Road, OX1 3RH \\ ${ }^{3}$ Department of Astronomy, California Institute of Technology, Pasadena, CA 91125, USA \\ ${ }^{4}$ Department of Astronomy, Yonsei University, Seoul 120-749, Republic of Korea \\ ${ }^{5}$ Department of Astronomy, University of Virginia, Charlottesville, VA 22904-4325, USA \\ ${ }^{6}$ School of Earth and Space Exploration, Arizona State University, Tempe, AZ 85287-1404, \\ USA \\ ${ }^{7}$ Space Telescope Science Institute, 3700 San Martin Drive, Baltimore, MD 21218, USA \\ email: s.kaviraj@imperial.ac.uk
}

\begin{abstract}
We summarise recent progress in understanding the star formation activity in earlytype galaxies (ETGs), using recent studies that leverage photometry in the rest-frame ultraviolet (UV) wavelengths. While classically thought to be old, passively-evolving systems, recent UV studies have revealed widespread star formation in ETGs, with $\sim 20 \%$ of the stellar mass in today's ETGs forming at late epochs $(z<1)$. A strong correlation is found between the presence of morphological disturbances and blue UV colours, suggesting that the star formation is mergerdriven. However, the major merger rate at late epochs is far too low to satisfy the number of disturbed ETGs, indicating that minor mergers drive the star formation in these galaxies over the latter half of cosmic time. Together with the recent literature which suggests that minor mergers may drive the size evolution of massive ETGs, these results highlight the significant role of minor mergers in driving the evolution of massive galaxies in the low and intermediate-redshift Universe.
\end{abstract}

Keywords. galaxies: elliptical and lenticular, $\mathrm{cD}$, galaxies: formation, galaxies: evolution, galaxies: interactions, galaxies: high-redshift, ultraviolet: galaxies

\section{Introduction}

Massive early-type galaxies (ETGs) dominate the stellar mass density in the local Universe. Hosting over half the stars at the present day, they hold a unique record of the evolution of the visible Universe over cosmic time. In recent decades, observational astronomy has focussed largely on the optical wavelengths, and our understanding of ETG evolution has naturally been shaped by such studies. The optical properties of ETGs (e.g. red optical colours and high ratios of alpha elements to iron), motivated a long-standing classical model (termed 'monolithic collapse'), which postulated their formation in short, efficient bursts of star formation at high redshift $(z \gg 1)$ followed by purely passive evolution thereafter (e.g. Bower et al. 1992; Renzini 2006). However, monolithic collapse is difficult to reconcile with the standard hierarchical paradigm of galaxy evolution, in which merger-driven and quiescent star formation is expected to take place in galaxies over the lifetime of the Universe.

Indeed, it can be shown that the optical predictions of semi-analytical models based on the standard paradigm are virtually indistinguishable from those of monolithic collapse 


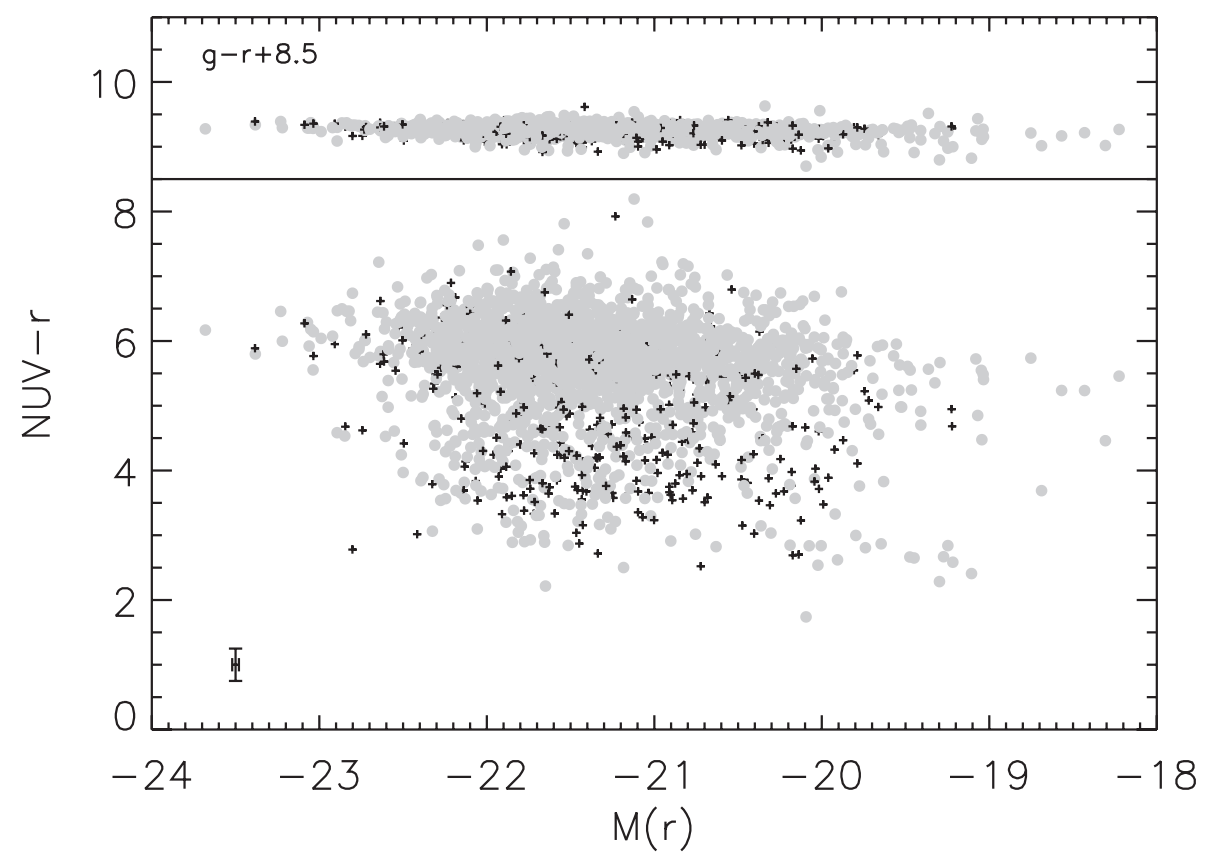

Figure 1. The optical (top panel) and the $N U V-r$ (bottom panel) colour-magnitude relations (CMRs) of our early-type galaxies. We intentionally show the optical CMR on the same scale as its $U V$ counterpart, to highlight the significant difference in their respective scatters. The small grey crosses represent galaxies which have Type II AGN. The maximum contamination in the UV flux due to Type II AGN is less than $15 \%$ (0.15 mag), which is much smaller than the observed scatter in the UV colours. The $N U V$ filter is centred at $2300 \AA$. This figure has been adapted from Kaviraj et al. 2007, ApJS, 173, 619.

(Kaviraj et al. 2005). This is because star formation in ETGs at late epochs $(z<1)$ is too weak to significantly impact the optical spectrum, implying that the optical wavelengths are not a good discriminant between the monolithic and hierarchical models. Accurately quantifying the star formation history of ETGs at late epochs requires a sensitive indicator of weak star formation, such as the rest-frame ultraviolet wavelengths (UV; 1200-3000 $\AA$ ), which is more than an order of magnitude more sensitive to star formation than the optical wavelengths.

\section{Persistent star formation in early-type galaxies since $z \sim 1$}

A series of recent studies has exploited rest-frame UV photometry of ETGs, to accurately measure the stellar mass growth in these galaxies since $z \sim 1$. Using data from NASA's GALEX UV space telescope, combined with optical data from the Sloan Digital Sky Survey (SDSS), Kaviraj et al. (2007) showed that, while the optical colours of local $(0<z<0.1)$ ETGs are indeed red, they exhibit almost 5 mags of spread in their rest-frame UV colours (Figure 1). Furthermore, the UV fluxes in the bluest $30 \%$ of ETGs are larger than the maximum UV flux that can be expected from old, extreme-horizontal-branch stars, which suggests that at least $30 \%$ of local ETGs contain unambiguous signatures of recent star formation. The mass fraction in young stars is of the order of a few percent and the luminosity-weighted average age of the young stellar populations is $~ 0.5$ Gyrs (Kaviraj et al. 2007). 
205

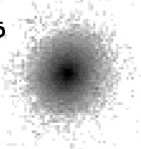

2575

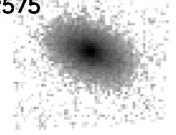

206

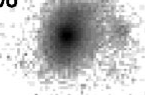

2528

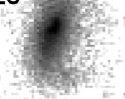

855

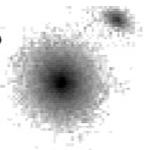

2721
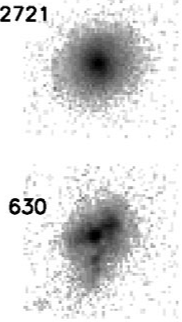

3023

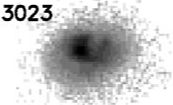

1618

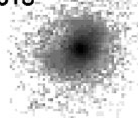

3132
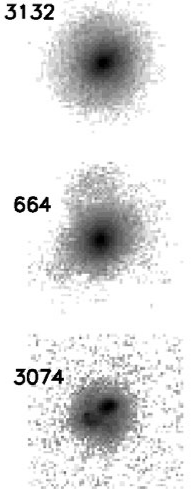

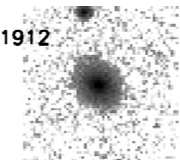

3156
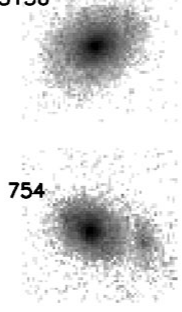

3146
2574
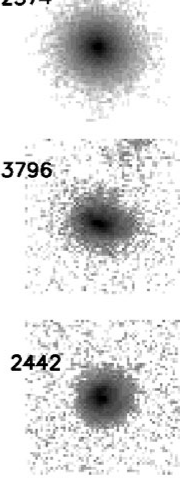

3166

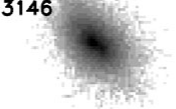

Figure 2. Examples of ETGs that are relaxed i.e. do not show any signs of morphological disturbances (rows 1 and 2) and ETGs that are morphologically disturbed (rows 3 and 4). This figure has been reproduced from Kaviraj et al. 2011, MNRAS, 411, 2148.

This result was subsequently consolidated by studying ETGs at intermediate redshift $(0.5<z<1)$. At these epochs the Universe is too young for the horizontal branch to be in place, making the UV a rather 'clean' indicator of recent star formation. Combining data from three intermediate-redshift surveys (MUSYC, COMBO-17 and GEMS) Kaviraj et al. (2008) demonstrated that the scatter in the UV colours of ETGs remained unchanged across the entire redshift range $0<z<1$. Together with the previous work using GALEX, this study concluded that ETGs of all luminosities form stars over the last 8 billion years, with massive (more luminous than $L_{*}$ ) ETGs forming $\sim 20 \%$ of their stellar mass after $z \sim 1$.

\section{Minor mergers as the principal driver of star formation in early-type galaxies}

While the studies described above established the presence of star formation in ETGs, ruling out the classical monolithic collapse model, they offered little insight into the sources of gas that drives this star formation. There are several potential sources of gas in ETGs, such as internal stellar mass loss, accretion from hot gas or ambient HI reservoirs and mergers. Stellar mass loss does not provide enough gas at the present day, ruling it out as the principal driver of star formation in local ETGs (Kaviraj et al. 2007). Indeed, the wider literature indicates that the interstellar medium of local ETGs may be external in origin. For example, results from the recent SAURON survey, which has performed integral-field-spectroscopy of local ETGs, indicate that the gas is often kinematically-decoupled from the stars (Sarzi et al. 2006). Measurements of dust masses in ETGs typically yield values that are in excess of the maximum value expected from stellar mass loss (e.g. Merluzzi 1998). Furthermore, more than $70 \%$ of ETGs appear morphologically disturbed in deep optical imaging (van Dokkum 2005).

To establish the role of mergers in driving the star formation in ETGs, Kaviraj et al. explored whether a correlation exists between the presence of morphological disturbances and blue UV colours. This is a difficult exercise to perform at low redshift, where imaging 


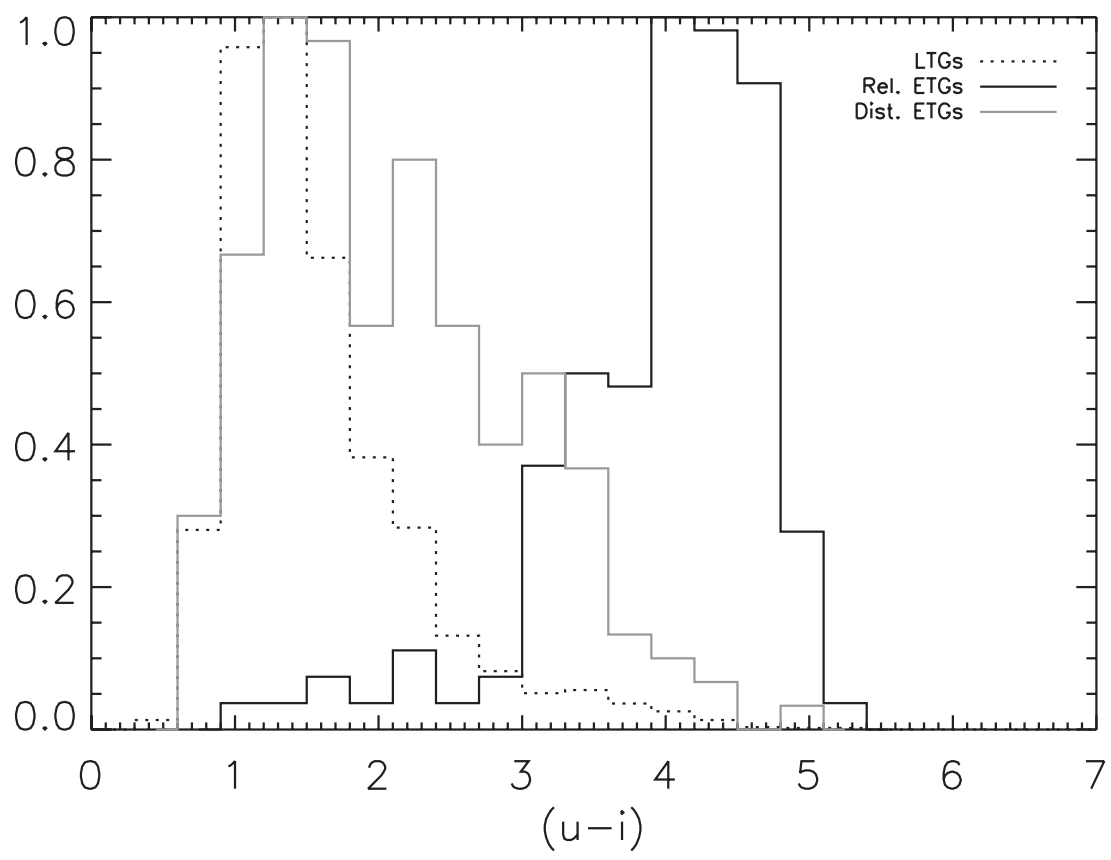

Figure 3. Rest-frame UV colour histograms for the relaxed ETG, disturbed ETG and late-type populations. Note that all histograms are normalised to 1 . This figure has been adapted from Kaviraj et al. 2011, MNRAS, 411, 2148.

from surveys such as the SDSS lack the resolution and depth to reveal faint morphological disturbances from e.g. minor mergers. However, this is possible using Hubble Space Telescope (HST) imaging at intermediate redshift. using surveys such as COSMOS. Kaviraj et al. found that ETGs at $z \sim 0.6$ that had blue UV colours were indeed highly likely to exhibit morphological disturbances (see Figures 2 and 3). However, the major merger rate at late epochs is several factors too low to satisfy the number of disturbed ETGs, indicating that minor mergers (with mass ratios between 1:4 and 1:10) drive the star formation in ETGs over the last 8 billion years. It is worth noting that the strong correlation between disturbed morphology and star formation also indicates that processes such as accretion from hot gas and ambient HI reservoirs are not responsible for triggering the star formation in these galaxies.

Although significant progress has been made over the past five years in deciphering the evolution of ETGs, only the broad outline of their star formation histories has been firmly established. While $\sim 80 \%$ of the stars in these galaxies are old, the remaining minority formed over the last 8 billion years via minor mergers between ETGs and small, gasrich companions. However, several outstanding questions remain. What are the detailed properties (mass ratios, satellite gas fractions, etc.) of the minor mergers that drive star formation in ETGs at late epochs? At what redshifts and through what processes (e.g. major mergers, cold streams) did the old, dominant stellar populations in today's ETGs form? The former can be answered using detailed spatially-resolved UV-optical analyses of local ETGs using new instruments such as HST's Wide Field Camera 3 (WFC3; e.g. Crockett et al. 2011), while the latter can be explored by studying newborn ETGs at the epoch of peak cosmic star formation, using new surveys of the high-redshift Universe that leverage the WFC3's near-infrared capabilities (e.g. CANDELS). The next five years offer us the tantalising possibility of accurately tracing the formation history of ETGs over 
$90 \%$ of the lifetime of the Universe and revolutionise our understanding of this important class of galaxy.

\section{References}

Bower R. G., Lucey J. R., \& Ellis R. 1992, MNRAS, 254, 589

Crockett R. M., Kaviraj S., Silk J. I., et al. 2011, ApJ, 727, 115

Kaviraj S., Devriendt J. E. G., Ferreras I., \& Yi S. K. 2005, MNRAS, 360, 60

Kaviraj S., Khochfar S., Schawinski K., et al. 2008, MNRAS, 388, 67

Kaviraj S., Schawinski K., Devriendt J. E. G., et al. 2007, ApJS, 173, 619

Merluzzi P. 1998, $A \& A, 338,807$

Renzini A. 2006, ARAA, 44, 141

Sarzi M., Falcón-Barroso J., Davies R. L., et al. 2006, MNRAS, 366, 1151

van Dokkum P. G. 2005, AJ, 130, 2647

\section{Discussion}

LEITHERER: Is there evidence for higher supernova rates in early-type galaxies with blue colours?

KAVIRAJ: Yes there is. Higher type Ic rates are found in ETGs with blue colours. Recent work also shows most type II rates are enhanced but sample sizes are quite small at the moment. 